\title{
Creating quantum correlations through local nonunitary memoryless channels
}

\author{
Francesco Ciccarello and Vittorio Giovannetti \\ NEST, Scuola Normale Superiore and Istituto Nanoscienze, Consiglio Nazionale delle Ricerche, Piazza dei Cavalieri 7, IT-56126 Pisa, Italy
}

(Received 8 June 2011; published 30 January 2012)

\begin{abstract}
We show that two qubits, initially in a fully classical state, can develop significant quantum correlations as measured by the quantum discord (QD) under the action of a local memoryless noise (specifically we consider the case of a Markovian amplitude-damping channel). This is analytically proven after deriving in a compact form the QD for the class of separable states involved in such a process. We provide a picture in the Bloch sphere that unambiguously highlights the physical mechanism behind the effect regardless of the specific measure of quantum correlations adopted.
\end{abstract}

DOI: 10.1103/PhysRevA.85.010102

PACS number(s): 03.65.Ud, 03.65.Yz, 03.65.Ta, 03.67.Mn

The existence of states where two or more systems are correlated in a way unattainable in classical physics ranks among the most puzzling and yet distinctive features of quantum mechanics. Such possibility is commonly pictured in terms of an extra amount of correlations, usually referred to as quantum correlations (QCs), which a multipartite system can possess in addition to those of a merely classical nature. Until recently, the scrutiny of QCs has been almost ubiquitously intertwined with investigations on entanglement [1] and the pivotal role that it plays in the area of quantum information processing [2]. A breakthrough yet occurred as soon as it was realized [3] that while classicality always entails separability the reverse is in general untrue (a state is entangled if it is nonseparable). Entanglement thereby is not the only form in which QCs can occur. Such finding brought about a widened perspective, which is currently prompting a growing number of researchers to advance the field along various lines. A prominent one is the quest for faithful easy-to-handle indicators of QCs [4,5]. Among those proposed so far, quantum discord (QD) [3] is having a considerable impact despite the fact that its explicit calculation is usually demanding even for two qubits (i.e., a pair of two-dimensional systems). Yet evidence of its ability to capture QCs not detected by entanglement has been supplied in various frameworks such as one-qubit quantum computation (even experimentally) [6] and quantum phase transitions [11].

Another major concern that soon arose is to assess how QCs, according to such a novel paradigm, are affected by nonunitary dynamics. These typically stem from the interaction with an environment, a process where entanglement is extremely fragile in most cases [1,2]. In contrast, QD was proven to be in general quite resilient to such dynamics and, strikingly, in some cases even fully insensitive over long stages [7]. From a reverse perspective, it was shown that the quantum noise arising from a common bath can create QCs initially fully absent [8], a phenomenon well known to occur for entanglement also [9]. Moreover, preexisting QCs can exhibit an increasing behavior at some stages of their time evolution in the presence of non-Markovian local channels, as recently demonstrated for both entanglement and QD [10]. In such instances, the increase of QCs stems either from the ability of a common memoryless reservoir to mediate an effective interaction or as a memory effect of local environments. The question is now raised: Can the interaction with a bath which is both local and memoryless enhance QCs? The answer is well known to be negative for entanglement, which cannot grow under any local quantum maps [1]. As for the full amount of QCs, however, the issue is not as trivial since as stressed above even separable states may feature some quantumness [3]. As far as local unitaries are concerned, though, QC measures, such as QD, cannot increase [3-5]. In some respects, this conclusion might be expected to be strengthened with noisy local operations. However, QCs without entanglement can arise under a local nonunitary operation owing to its ability to map orthogonal into nonorthogonal states [5]. In this work, we present a simple paradigmatic process, where entanglement is absent throughout, which clearly testifies that QCs can even be entirely created (or increased) solely via the interaction with a bath which is both local and memoryless. We provide rigorous and comprehensive insight into such an effect in a way that makes transparent the underlying physical mechanism. Although we focus on QD, it will become clear that the essential physical effect takes place regardless of the specific measure of QCs used.

To begin with, we briefly recall the definition of QD. Given two systems $A$ and $B$ in a state $\rho$, this measures the discrepancy between the mutual information $\mathcal{I}$ and the classical correlations $\mathcal{C}$ associated with $\rho$ [3]. A local measurement on $B$ in a given orthonormal basis can be specified by a complete set of projectors $\left\{B_{k}\right\}$, where $k$ indexes a possible outcome. If $k$ is recorded with probability $p_{k}=\operatorname{Tr}\left[B_{k} \rho B_{k}\right]$ the overall system collapses onto the (normalized) state $\rho_{k}=\left(B_{k} \rho B_{k}\right) / p_{k}$. Then the QD $\mathcal{D}^{\leftarrow}$ can be expressed as [3]

$$
\mathcal{D}^{\leftarrow}(\rho)=S\left(\rho_{B}\right)-S(\rho)+\min _{\left\{B_{k}\right\}} \sum_{k} p_{k} S\left(\rho_{k}\right) .
$$

In Eq. (2), $\rho_{B}=\operatorname{Tr}_{A} \rho$ is the reduced density operator that describes the state of $B, S(\sigma)=-\operatorname{Tr}\left(\sigma \log _{2} \sigma\right)$ is the Von Neumann entropy of an arbitrary state $\sigma$, and the infimum in the last term is evaluated over all the possible sets $\left\{B_{k}\right\}$. As for the $\mathrm{QD} \mathcal{D}^{\rightarrow}$ involving measurements on $A$ with associated projectors $\left\{A_{k}\right\}$, this is obtained from Eq. (1) through replacement of $B$ with $A$. In general, $\mathcal{D}^{\leftarrow} \neq \mathcal{D}^{\rightarrow}$ [3].

Specifically, in the process under study $A$ and $B$ are two qubits and the only involved states have the separable form

$$
\rho=\frac{1}{2}\left(|0\rangle_{A}\left\langle 0\left|\otimes \tau_{0 B}+\right| 1\right\rangle_{A}\langle 1| \otimes \tau_{1 B}\right)
$$



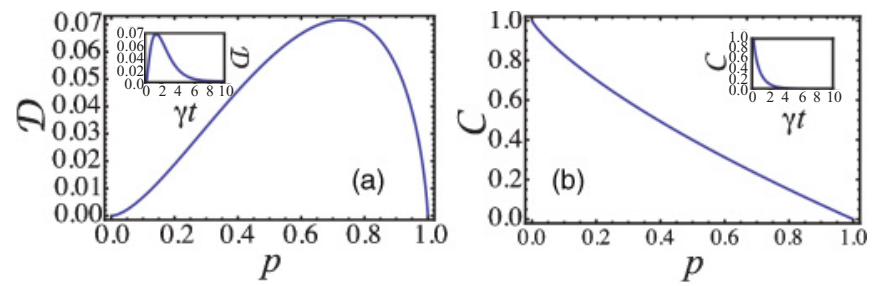

FIG. 1. (Color online) Behavior under a local amplitude-damping channel of (a) discord $\mathcal{D}$ and (b) classical correlations $\mathcal{C}$ against $p$. In each panel, the inset shows the corresponding behavior expressed as a function of the rescaled time $\gamma t$, where $p=1-e^{-\gamma t}$.

where $\tau_{0(1)}$ are generic density matrices, while the orthonormal set of vectors $\{|0\rangle,|1\rangle\}$ is the usual local computational basis. The state Eq. (2) are commonly dubbed as classical-quantum states owing to $\mathcal{D} \rightarrow=0$ as is immediate to check [12], while in general $\mathcal{D}^{\leftarrow} \neq 0$. We will thereby set $\mathcal{D} \equiv \mathcal{D}^{\leftarrow}$ henceforth.

Consider now the initial state

$$
\rho_{0}=\frac{1}{2}\left(|0\rangle_{A}\langle 0|\otimes|+\rangle_{B}\langle+|+| 1\rangle_{A}\langle 1|\otimes|-\rangle_{B}\langle-|\right),
$$

i.e., in the light of Eq. (2) $\tau_{0}=|+\rangle\langle+|$ and $\tau_{1}=|-\rangle\langle-|$, where $| \pm\rangle=(|0\rangle \pm|1\rangle) / \sqrt{2}$. Although manifestly correlated, state Eq. (3) is fully classical since it is diagonal in the basis resulting from the tensor product between $\left\{|0\rangle_{A},|1\rangle_{A}\right\}$ and $\left\{|+\rangle_{B},|-\rangle_{B}\right\}$, namely two local orthogonal bases [13]. Thus $\mathcal{D}\left(\rho_{0}\right)=0$. Assume now that, while $A$ is well protected from the external environment, $B$ is in contact with a dissipative Markovian bath. In such a case, the system dynamics as a function of time $t$ is fully described by an amplitude-damping channel [2]. This has an associated quantum map $\mathcal{E}_{p}$ that transforms state $\rho_{0}$ according to $\mathcal{E}_{p}\left(\rho_{0}\right)=E_{0} \rho_{0} E_{0}^{\dagger}+E_{1} \rho_{0} E_{1}^{\dagger}$, where $E_{0}=|0\rangle_{B}\langle 0|+\sqrt{1-p}| 1\rangle_{B}\langle 1|$ and $E_{1}=\sqrt{p}|0\rangle_{B}\langle 1|$ are the associated Kraus operators, while $p$ is a probability that grows with time $t$ according to $p=1-e^{-\gamma t}$ ( $\gamma$ is a relaxation rate). As anticipated, $\mathcal{E}_{p}\left(\rho_{0}\right)$ evidently belongs to class Eq. (2) for any $p$ (i.e., $\forall t$ ) since $E_{0}$ and $E_{1}$ act on $B$ only (hence entanglement never appears throughout). In Fig. 1(a), we plot $\mathcal{D}$ [cf. Eq. (1)] against $p$ and the rescaled time $\gamma t$ as resulting from numerical evaluation [14]. $\mathcal{D}(p)$, which is initially null, as discussed above, at a first stage grows and eventually decays to zero as $p \rightarrow 1$, i.e., for $t \gg \gamma^{-1}$. This marks a profound difference between QD and entanglement in that a local nonunitary and memoryless channel is able to create QCs previously fully absent. Remarkably, the dissipative dynamics is merely detrimental to the classical correlations $\mathcal{C}=\mathcal{I}-\mathcal{D}$ [3], where $\mathcal{I}=S\left(\rho_{A}\right)+S\left(\rho_{B}\right)-S(\rho)$ is the mutual information [2]. $\mathcal{C}$ indeed exhibits a monotonic decay vanishing for $p \rightarrow 1$, i.e., $\gamma t \rightarrow \infty$, as shown in Fig. 1(b).

In the following, we make this result rigorous by analytically deriving $\mathcal{D}(p)$ and $\mathcal{C}(p)$ so as to reproduce Fig. 1. To this aim, we first explicitly derive in a compact form the QD of state Eq. (2) when $\tau_{0}$ and $\tau_{1}$ have the same purity. Next, we present a picture in terms of trajectories in the Bloch sphere, clearly highlighting the physical mechanism that causes QD to necessarily grow in the present process.

Among state Eq. (2) a prominent instance is the resource state for the Bennett 1992 (B92) quantum cryptography protocol [2], which reads $\rho_{\mathrm{B} 92}=\frac{1}{2}\left(|0\rangle_{A}\langle 0|\otimes| 0\rangle_{B}\langle 0|+| 1\rangle_{A}\langle 1| \otimes\right.$
$\left.|+\rangle_{B}\langle+|\right)$. Similarly to the popular Bennett-Brassard 1984 (BB84) protocol [2] the above is among those quantum protocols where the exploited quantum resource is not entanglement, which is is fully absent. Rather, it harnesses one-way QCs stemming from the nondistinguishability of states $|0\rangle$ and $|+\rangle$ [13]. Later on, we will indeed show that $\rho_{\mathrm{B} 92}$ possesses the maximum allowed QD within family Eq. (2) with $\tau_{0}$ and $\tau_{1}$ having equal purities. Also, states such as Eq. (2) can allow for quantum locking (see Ref. [15] and references therein). To our knowledge, the literature lacks explicit formulas for the QD of Eq. (2). We will thus carry out an ab initio calculation.

To this aim, our first step is to express the single-qubit states $\tau_{0}$ and $\tau_{1}$ in Eq. (2) through the Bloch-sphere representation as $\tau_{i}=\left(\mathbb{1}+\mathbf{s}_{i} \cdot \boldsymbol{\sigma}\right) / 2$, where $\mathbb{1}$ and $\boldsymbol{\sigma}=\left\{\sigma_{1}, \sigma_{2}, \sigma_{3}\right\}$ are the usual identity and Pauli operators, respectively, while $\mathbf{s}_{i}$ is the Bloch vector corresponding to $\tau_{i}(i=0,1)$. Without loss of generality we can assume that $s_{0 x}=s_{0 y}=0, s_{0 z} \equiv s_{0}$, and $s_{1 y}=0$; i.e., in the Bloch sphere $\mathbf{s}_{0}$ and $\mathbf{s}_{1}$ lie on the $x-z$ plane with $\mathbf{s}_{0}$ along the $z$ axis $\left(s_{i}=\left|\mathbf{s}_{i}\right| \leqslant 1\right)$. Indeed, one can reduce the problem to such a case by applying a suitable single-qubit rotation, which cannot affect the QD like any local unitary operation [3]. Using this along with $|0\rangle\langle 0|=\left(\mathbb{1}+\sigma_{3}\right) / 2$ and $|1\rangle\langle 1|=\left(\mathbb{1}-\sigma_{3}\right) / 2$, state Eq. (2) can be arranged as (from now on we drop subscripts $A$ and $B$ )

$$
\rho=\frac{1}{4}\left[\mathbb{1} \otimes \mathbb{1}+\mathbb{1} \otimes\left(a_{1} \sigma_{1}+a_{3} \sigma_{3}\right)+\sigma_{3} \otimes\left(b_{1} \sigma_{1}+b_{3} \sigma_{3}\right)\right],
$$

with

$$
\begin{aligned}
& a_{1}=-b_{1}=\frac{s_{1} \sin \varphi}{2}, \quad a_{3}=\frac{s_{0}+s_{1} \cos \varphi}{2}, \\
& b_{3}=\frac{s_{0}-s_{1} \cos \varphi}{2},
\end{aligned}
$$

where we have carried out the replacements $s_{1 x}=s_{1} \sin \varphi$, $s_{1 z}=s_{1} \cos \varphi\left(\varphi\right.$ is the angle between $\mathbf{s}_{0}$ and $\left.\mathbf{s}_{1}\right)$. Next, in the light of Eq. (1) we need to calculate how $\rho$ is transformed when a generic Von Neumann measurement is performed on system $B$, i.e., $\rho_{k}$, as well as the associated probability $p_{k}$. As in [16] we use the property that the projector corresponding to any such measurement can be expressed as $\mathbb{1} \otimes B_{k}$ with $B_{k}=V \Pi_{k} V^{\dagger}$, where $\Pi_{k}=|k\rangle\langle k|(k=0,1)$ and $V$ is a generic one-qubit unitary. We thus expand $p_{k} \rho_{k}=\left(\mathbb{1} \otimes B_{k}\right) \rho\left(\mathbb{1} \otimes B_{k}\right)$ as

$p_{k} \rho_{k}=(\mathbb{1} \otimes V)\left(\mathbb{1} \otimes \Pi_{k}\right)\left(\mathbb{1} \otimes V^{\dagger}\right) \rho(\mathbb{1} \otimes V)\left(\mathbb{1} \otimes \Pi_{k}\right)\left(\mathbb{1} \otimes V^{\dagger}\right)$.

The unitary $V^{\dagger}$ transforms each Pauli matrix $\sigma_{i}$ according to $V^{\dagger} \sigma_{i} V=\sum_{j=1}^{3} v_{i j} \sigma_{j}$, where $v_{i j}$ are real numbers satisfying the constraint $v_{1 j}^{2}+v_{2 j}^{2}+v_{3 j}^{2}=1$, for any $j=1,2,3$. Also, for $k=0,1 \Pi_{k} \sigma_{1} \Pi_{k}=\Pi_{k} \sigma_{2} \Pi_{k}=\mathbf{0}$ while $\Pi_{k} \sigma_{3} \Pi_{k}=f_{k} \Pi_{k}$, where $f_{0}=1$ and $f_{1}=-1$. This along with Eq. (4) yields Eq. (6) in the simpler form

$$
\begin{aligned}
p_{k} \rho_{k} & =(\mathbb{1} \otimes V)\left[\frac{\mu_{k} \mathbb{1}+v_{k} \sigma_{3}}{4} \otimes \Pi_{k}\right]\left(\mathbb{1} \otimes V^{\dagger}\right) \\
& =\frac{\mu_{k} \mathbb{1}+v_{k} \sigma_{3}}{4} \otimes\left(V \Pi_{k} V^{\dagger}\right)
\end{aligned}
$$


where

$\mu_{k}=1+f_{k}\left(a_{1} v_{13}+a_{3} v_{33}\right), \quad v_{k}=f_{k}\left(b_{1} v_{13}+b_{3} v_{33}\right)$.

As $V \Pi_{k} V^{\dagger}$ represents a pure state and each Pauli matrix is traceless it is immediately checked that $p_{k}=\mu_{k} / 2$. Therefore, we obtain that $\rho_{k}=\left(\mathbb{1}+v_{k} / \mu_{k} \sigma_{3}\right) / 2 \otimes\left(V \Pi_{k} V\right)$, whose eigenvalues are $\left(1 \pm v_{k} / \mu_{k}\right) / 2$ (each twofold degenerate). The quantity to minimize entering the last term of Eq. (1) thus reads

$\delta\left(v_{13}, v_{33}\right)=\sum_{k=0,1} p_{k} S\left(\rho_{k}\right)=\sum_{k=0,1} \frac{\mu_{k}}{2} h\left(\frac{1 \pm v_{k} / \mu_{k}}{2}\right)$,

where $h(x)=-x \log _{2} x-(1-x) \log _{2}(1-x)$ is the binary Shannon entropy function and on the left-hand side we have highlighted the dependence on variables $v_{13}$ and $v_{33}$. The identity $v_{13}^{2}+v_{23}^{2}+v_{33}^{2}=1$ (see above) yields that $\left\{v_{13}, v_{33}\right\}$ must fulfill $v_{13}^{2}+v_{33}^{2}=1-v_{23}^{2} \leqslant 1$; i.e., they belong to the unit circle. To work out the infimum of $\delta$ we accomplish the linear transformation $x=a_{1} v_{13}+a_{3} v_{33}$ and $y=b_{1} v_{13}+$ $b_{3} v_{33}$. This way, Eq. (8) now becomes a universal function of $x$ and $y$, which we call $\tilde{\delta}(x, y)$; i.e., it no longer depends on parameters $\left\{a_{i}, b_{i}\right\}$ specifying states $\tau_{0}$ and $\tau_{1}$ [cf. Eq. (5)].

In Fig. 2(a) we plot $\tilde{\delta}$ against $x$ and $y$. Note that its actual domain of definition is the square having side length $1 / \sqrt{2}$ and vertices at points $( \pm 1,0)$ and $(0, \pm 1)$ [this is because of the logarithms of $1 \pm f_{k} y /\left(1+f_{k} x\right)$; see Eqs. (7) and (8)]. Also, $\tilde{\delta}$ is an even function of $x(y)$ for any set value of $y(x)$ as is also clear from its dependance on $x$ and $y$ through $\mu_{k}$ and $v_{k}$ [see Eqs. (7) and (8)]. It takes value 1 for $x=y=0$ and decreases as the distance from the origin grows. Its concavity is minimum along the $x$ axis, where the function is fully flat, and maximum along the $y$ axis [see the sail-like shape in Fig. 2(a)]. As for the domain $\tilde{D}$ within which Eq. (8) is to be minimized, i.e., the region in the new reference frame corresponding to the unit circle $v_{13}^{2}+v_{33}^{2} \leqslant 1$ (see above), it is immediately checked

(a)

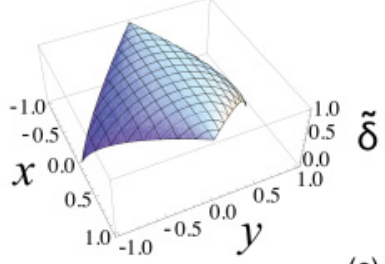

(b)

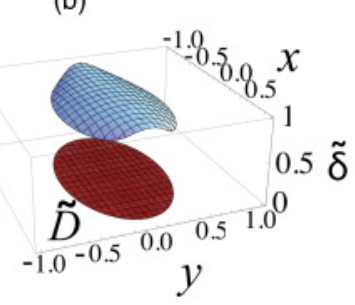

(c)

FIG. 2. (Color online) (a) Function $\tilde{\delta}(x, y)$ for $|x| \leqslant 1$ and $|y| \leqslant$ 1. (b) and (c) Function $\tilde{\delta}(x, y)$ for $(x, y) \in \tilde{D}$ as given by Eq. (9) when $s_{0}=s_{1}=1$ and (b) $\varphi=\pi / 3$ and (c) $\varphi=2 \pi / 3$. In either case, the red region on the $x-y$ plane represents the corresponding $\tilde{D}$. through the inverse transformation that this is an elliptic region given by

$$
\begin{aligned}
& \tilde{D}=\left\{x, y: A x^{2}+2 B x y+C y^{2} \leqslant 1\right\}, \\
& A=\left(\frac{\left|\mathbf{s}_{\mathbf{0}}-\mathbf{s}_{\mathbf{1}}\right|}{s_{0} s_{1} \sin \varphi}\right)^{2}, \quad B=\frac{s_{0}^{2}-s_{1}^{2}}{\left(s_{0} s_{1} \sin \varphi\right)^{2}}, \\
& C=\left(\frac{\left|\mathbf{s}_{\mathbf{0}}+\mathbf{s}_{\mathbf{1}}\right|}{s_{0} s_{1} \sin \varphi}\right)^{2} .
\end{aligned}
$$

As is evident from Fig. 2(a), owing to the concavity of $\tilde{\delta}$ its infimum under constraint Eq. (9) necessarily lies on the boundary of $\tilde{D}$, i.e., the ellipse obtained from Eq. (9) by turning the inequality into an identity. While such an ellipse is centered at $x=y=0$, its own axes in general do not coincide with those defining the reference frame. Yet, in the case that $B=0$ [cf. Eqs. (9) and (10)] the ellipse is not rotated with respect to the $x$ and $y$ axis. This circumstance physically occurs when $\tau_{0}$ and $\tau_{1}$ have the same purity, i.e., $s_{0}=s_{1}$ [see Eq. (10)]. Henceforth, we will focus on such a case, which is enough for the scope of the present work and allows for a prompt analytical derivation of the infimum of Eq. (8) under constraint Eq. (9). Hence, by setting $s_{0}=s_{1}=s$ coefficient Eqs. (10) become $A=1 / r_{x}^{2}, B=0, C=1 / r_{y}^{2}$, where $r_{x}=s|\sin (\varphi / 2)|$ and $r_{y}=s|\cos (\varphi / 2)|$ are the ellipse semiaxis lengths along the $x$ and $y$ axis, respectively. Now, because of the shape of $\tilde{\delta}(x, y)$ [see Fig. 2(a)], when the main axes and the ellipse's own ones are collinear $\tilde{\delta}(x, y)$ takes its minimum at the points $\left(0, \pm r_{y}\right)$. This is evident from Figs. 2(b) and 2(c), where we plot the restriction of $\tilde{\delta}$ to region $\tilde{D}$ in two paradigmatic cases. The one in Fig. 2(b) [Fig. 2(c)] is such that $r_{x}>r_{y}\left(r_{x}<r_{y}\right)$. The minimum of Eq. (8) within region Eq. (9), i.e., the last term in Eq. (1), thus reads

$$
\begin{aligned}
\min _{\tilde{D}} \tilde{\delta}(x, y) & =\tilde{\delta}\left(0, r_{y}\right)=\tilde{\delta}\left(0, s\left|\cos \frac{\varphi}{2}\right|\right) \\
& =h\left[\frac{1+s|\sin \varphi / 2|}{2}\right],
\end{aligned}
$$

where we used the fact that $\mu_{k}=1+f_{k} x$ and $v_{k}=f_{k} y$ [cf. Eqs. (7) and (8)].

As for $S\left(\rho_{B}\right)$, the trace over $A$ of Eq. (4) is obtained as $\rho_{B}=\left(\mathbb{1}+a_{1} \sigma_{1}+a_{3} \sigma_{3}\right) / 2$, whose eigenvalues with the help of Eq. (5) are found as $(1 \pm s|\cos \varphi / 2|) / 2$. Hence, $S\left(\rho_{B}\right)=h[(1+s|\cos \varphi / 2|) / 2]$. Using again Eqs. (4) and (5), the eigenvalues of $\rho$ are calculated as $(1 \pm s) / 4$, each being two-fold degenerate yielding that $S(\rho)=1+h[(1+s) / 2]$. Using these results along with Eq. (11) in the light of Eq. (1), we find the QD of any state Eq. (2) such that $\tau_{0}$ and $\tau_{1}$ have the same Bloch-vector length $s$ in the compact form

$$
\begin{aligned}
\mathcal{D}= & h\left[\frac{1+s|\cos \varphi / 2|}{2}\right]+h\left[\frac{1+s|\sin \varphi / 2|}{2}\right] \\
& -h\left[\frac{1+s}{2}\right]-1 .
\end{aligned}
$$

As for the classical correlations $\mathcal{C}=\mathcal{I}-\mathcal{D}$, using the fact that $S\left(\rho_{A}\right)=S\left(\mathbb{1}_{A} / 2\right)=1$ we find that

$$
\mathcal{C}=1-h\left[\frac{1+s|\sin \varphi / 2|}{2}\right] \text {. }
$$


(a)

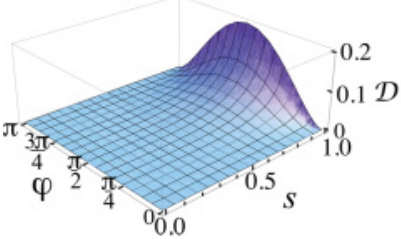

(b)

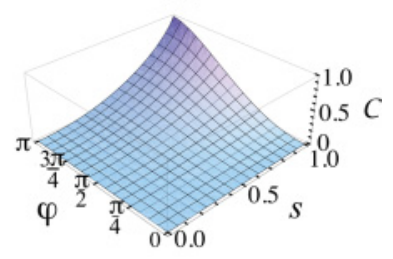

FIG. 3. (Color online) (a) $\mathcal{D}$ and (b) $\mathcal{C}$ vs $s$ and $\varphi$.

In Fig. 3 we plot $\mathcal{D}$ (a) and $\mathcal{C}$ (b) versus $s$ and $\varphi$ as given by Eqs. (12) and (13). For a given $s$, i.e., for fixed purity, $\mathcal{D}$ solely depends on $|\varphi-\pi / 2|$ exhibiting its maximum value when $\varphi=\pi / 2$. As $|\varphi-\pi / 2|$ grows the QD progressively decreases until it vanishes at $\varphi=0, \pi$. On the other hand, for a given $\varphi, \mathcal{D}$ increases with the purity at a rate that grows as $\varphi$ approaches $\pi / 2$. Within the present class of states, the QD thus takes its maximum value $\mathcal{D}_{\max } \simeq 0.202$ [about three times larger than the maximum value attained in Fig. 1(a)] for $s=1$ and $\varphi=\pi / 2$, which correspond to the B92 resource state $\rho_{\mathrm{B} 92}$ introduced previously. This feature is in accordance with Ref. [17] (where a different QC measure was used). As for $\mathcal{C}$, Fig. 3(b) shows that this is maximum for $s=1$ and $\varphi=\pi$ and decreases when either of such two parameters is reduced.

We are now in a position to provide a comprehensive explanation for the effect in Fig. 1. Using the abovediscussed Kraus decomposition of $\mathcal{E}_{p}$ it is straightforwardly found that $\mathcal{E}_{p}(| \pm\rangle\langle \pm|)=\left(\mathbb{1} \pm \sqrt{1-p} \sigma_{1}+p \sigma_{3}\right) / 2$. Hence, $s_{0 x}=-s_{1 x}=\sqrt{1-p}$, while $s_{0 z}=s_{1 z}=p$ (note that this immediately shows that $s_{0} \equiv s_{1}=s$ for any $p$ ). Using this, we end up with

$s(p)=\sqrt{1+p(p-1)}, \quad \varphi(p)=\pi-2 \arctan (p / \sqrt{1-p})$.

By replacing Eq. (14) in Eqs. (12) and (13), the functions $\mathcal{D}(p)$ and $\mathcal{C}(p)$ so obtained reproduce the plots in Fig. 1.

To shed light on the physical mechanism behind the effect, in Fig. 4 we plot on the $x-z$ plane of the Bloch sphere the parabolic trajectories of the Bloch vectors corresponding to $\mathcal{E}_{p}(| \pm\rangle\langle \pm|)$. Initially, the two vectors have unit lengths pointing toward opposite directions of the $x$ axis. Hence, $s=1$ and $\varphi=\pi$, which yields that $\mathcal{D}=0$ and $\mathcal{C}=1$ [cf. Eqs. (12) and (13) and Fig. 3]. When $p=1$, i.e., for $\gamma t \gg 1$, either state is mapped onto $|0\rangle\langle 0|$, i.e., the North Pole, which gives us that $s=1, \varphi=0$ and thereby $\mathcal{D}=\mathcal{C}=0$ [Eq. (2) indeed shows that the state becomes evidently uncorrelated]. At the intermediate stage, map $\mathcal{E}_{p}$ acts in a way that the two Bloch vectors move along symmetrical parabolic trajectories (see Fig. 4) yielding that, in

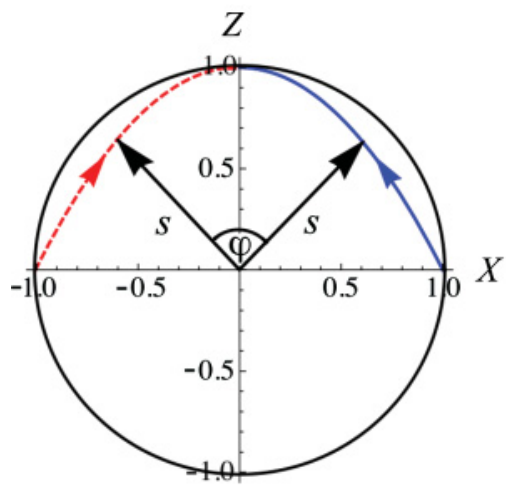

FIG. 4. (Color online) Trajectories of $\mathcal{E}_{p}(|+\rangle\langle+|)$ (blue solid line) and $\mathcal{E}_{p}(|-\rangle\langle-|)$ (red dashed line) on the $X-Z$ plane of the Bloch sphere.

particular, $0<\varphi<\pi$. On the other hand, the purity shows a dip with $s$ taking its minimum $s_{\min }=\sqrt{3} / 2$ for $p=1 / 2$ in accordance with Eq. (14). In the light of Eq. (12) and Fig. 3(a), this necessarily brings about that $\mathcal{D}>0$ since $0<\varphi<\pi$ and the Bloch vector length, although shrinking to some extent, keeps finite throughout. This clarifies why discord must necessarily be created in the course of the process. In essence, the local dissipative channel transforms $|+\rangle\langle+|$ and $|-\rangle\langle-|$ so as to reduce their distinguishability, which unavoidably gives rise to QCs without entanglement [3,13]. Note that the nonunitarity is crucial since distinguishability as measured by the scalar product is unaffected by any unitary [5,18]. Also, note that based on the definition of classically correlated states [13] the above reasoning shows that QCs will be surely created regardless of the specific measure chosen to quantify them.

In summary, in this paper we asked whether QCs can develop as a result of local nonunitary dynamics, an unattainable phenomenon with entanglement. After deriving the QD for the class of involved states, we analytically proved that this indeed can occur for two qubits, initially in a fully classical state, under a local memoryless amplitude-damping channel. Also, we showed that the mechanism behind the QCs' birth can be readily grasped in the Bloch-sphere picture.

All these phenomena are arguably not restricted to qubits. The generalization to continuous-variable systems in a way that local bosonic Gaussian maps now play the role of the amplitude-damping channel is under ongoing investigations.

Note added. Recently, we became aware of a related manuscript by Campbell et al. [19].

We thank R. Fazio and M. Paternostro for comments and acknowledge support from Fondo per gli Investimenti della Ricerca di Base (FIRB) IDEAS through project RBID08B3FM.
[1] R. Horodecki et al., Rev. Mod. Phys. 81, 865 (2009).

[2] M. A. Nielsen and I. L. Chuang, Quantum Computation and Quantum Information (Cambridge University Press, Cambridge, 2000).
[3] L. Henderson and V. Vedral, J. Phys. A 34, 6899 (2001); H. Ollivier and W. H. Zurek, Phys. Rev. Lett. 88, 017901 (2001).

[4] J. Oppenheim, M. Horodecki, P. Horodecki, and R. Horodecki, Phys. Rev. Lett. 89, 180402 (2002); B. Groisman, S. Popescu, 
and A. Winter, Phys. Rev. A 72, 032317 (2005); S. Luo, ibid. 77, 022301 (2008); B. Dakić, V. Vedral, and C. Brukner, Phys. Rev. Lett. 105, 190502 (2010); D. Girolami, M. Paternostro, and G. Adesso, e-print arXiv:1008.4136.

[5] K. Modi, T. Paterek, W. Son, V. Vedral, and M. Williamson, Phys. Rev. Lett. 104, 080501 (2010).

[6] A. Datta, A. Shaji, and C. M. Caves, Phys. Rev. Lett. 100, 050502 (2008); B. P. Lanyon, M. Barbieri, M. P. Almeida, and A. G. White, ibid. 101, 200501 (2008).

[7] T. Werlang, S. Souza, F. F. Fanchini, and C. J. VillasBoas, Phys. Rev. A 80, 024103 (2009); J. Maziero, L. C. Celeri, R. M. Serra, and V. Vedral, ibid. 80, 044102 (2009); L. Mazzola, J. Piilo, and S. Maniscalco, Phys. Rev. Lett. 104, 200401 (2010).

[8] J.-B. Yuan, L.-M. Kuang, and J.-Q. Liao, J. Phys. B At. Mol. Opt. Phys. 43, 165503 (2010); F. Altintas and R. Eryigit, e-print arXiv:1105.2222 [quant-ph].

[9] D. Braun, Phys. Rev. Lett. 89, 277901 (2002); F. Benatti, R. Floreanini, and M. Piani, ibid. 91, 070402 (2003); T. S. Cubitt, F. Verstraete, W. Dur, and J. I. Cirac, ibid. 91, 037902 (2003).

[10] B. Bellomo, R. Lo Franco, and G. Compagno, Phys. Rev. Lett. 99, 160502 (2007); F. F. Fanchini, T. Werlang, C. A. Brasil, L. G. E. Arruda, and A. O. Caldeira, Phys. Rev. A 81, 052107 (2010).

[11] M. S. Sarandy, Phys. Rev. A 80, 022108 (2009).
[12] By definition, $\mathcal{D}^{\rightarrow} \geqslant 0$ [3]. To prove that $\mathcal{D}^{\rightarrow} \equiv 0$ it suffices to select $A_{0}=|0\rangle_{A}\langle 0|$ and $A_{1}=|1\rangle_{A}\langle 1|$ as projectors. Then $p_{0}=p_{1}=1 / 2$ and $\sum_{k} p_{k} S\left(\rho_{k}\right)=1 / 2 \sum_{k=0,1} S\left(\sigma_{k}\right)$. Also, $S\left(\rho_{A}\right)=S\left(\mathbb{1}_{A} / 2\right)=1$ while $S(\rho)=1+1 / 2 \sum_{k} S\left(\sigma_{k}\right)$. Use of Eq. (1) then gives $\mathcal{D}^{\rightarrow}=0$.

[13] B. Groisman, D. Kenigsberg, and T. Mor, e-print arXiv:quant-ph/0703103.

[14] We used the fact that any set of one-qubit orthonormal projectors can be expressed as $\left\{M_{k}=\left|\Psi_{k}\right\rangle\left\langle\Psi_{k}\right|\right\} \quad(k=$ $0,1)$ with $\left|\Psi_{0}\right\rangle=\left(\cos \vartheta|0\rangle+e^{i \phi} \sin \vartheta|1\rangle\right) / \sqrt{2}$ and $\left|\Psi_{1}\right\rangle=$ $\left(e^{-i \phi} \sin \vartheta|0\rangle-\cos \vartheta|1\rangle\right) / \sqrt{2}$, where $\vartheta \in[0, \pi / 2]$ and $\phi \in$ $[0,2 \pi]$. We then discretized $\mathcal{D}$ through a mesh of $\vartheta$ and $\phi$, took its minimum value throughout, and eventually checked its stability against the number of grid points.

[15] S. Boixo et al., e-print arXiv:1105.2768.

[16] S. Luo, Phys. Rev. A 77, 042303 (2008); M. Ali, A. R. P. Rau, and G. Alber, ibid. 81, 042105 (2010).

[17] S. Gharibian et al., Int. J. Quantum Info. 9, 1701 (2011).

[18] The task to spoil the distinguishability of $\{| \pm\rangle\langle \pm|\}$ giving rise to QCs could also be accomplished via an engineered local quantum operation on $B$ (see Ref. [5] and references therein). It is quite remarkable, though, that a spontaneously occurring dissipative dynamics, typically regarded as undesirable for the sake of quantum information processing, can be effective also.

[19] S. Campbell et al., Phys. Rev. A 84, 052316 (2011). 